To list an event in the Calendar, contact J. Dininny, Materials Research Society, 9800 McKnight Road, Pittsburgh, PA 15237; (412) 367-3036; $\operatorname{tax}(412)$ 367-4373

MIRIS identifies meetings sponsored or co-sponsored by the Materials Research Society; boldface type without the logo identifies MRS-endorsed meetings.

SEe MRS BULLETIN VOI. XV No. 10 tor Calendar events from November 1 through November 30, 1990

\section{DECEMBER 1990}

$6-7$

Workshop on Fundamentals of Carbon/Carbon

National Institute of Standards and Technology

T. Grice, NIST, Gaithersburg, MD 20899; (301) 975-2775

$9-14$

Influence of Japanese/American Cultures on Technologica Innovation in Advanced Materials (Conterence)

Tuscon, Arizona

W.D. Kingery, Univ. of Arizona, Program on Culture, Science and Technology, Room 338E, Building

12. Tuscon, AZ 85721; tax (602) $621-8117$

$16-20$

2nd international Topical Workshop on Advances in SiliconBased Polymer Science

Oahy, Hawail

D. Morill, VPI \& SU, 1103 CRB, Blacksburg, VA 24061-0212; (703) 231-3029

\section{7}

Workshop on Laser Ablation of Superconductors London, England Superconductor Workshop Secretariat, Electronic \& Electrical Engineering, University College London, Torrington Place, London WC1E 7JE, UK; 4471380-7304; $\operatorname{tax} 44-71-387-4350$

\section{JANUARY 1991}

13-18

Mechanical Fatigue in Advanced Materials

Santa Barbara, CA

R.J. Yacyshyn, Engineening Foundation, 345 E. 47th St., New York, NY 10017; (212) 705-7835
6-18

2nd ISSP Intemational Symposium on Physics and Chemistry of Oxide Superconductors

(PCOS '91)

Tokyo, Japan

Y. Lye, Secretariat, PCOS '91, Institute for Solid State Physics. University of Tokyo, Roppongi,

Minato-ku, Tokyo 106, Japan; 3

478-6811; $\operatorname{tax} 3-401-5160$

\section{8-31}

Intemational Conference on Condensed Matter Physics and Applications

Isa Town, Bahrain

S. Aljishi, Univ of Bahrain,

Physics Dept., P.O. Box 32038,

Isa Town, Bahrain; (973) 681234 $\operatorname{tax}(973) 681465$

$29-1$

PCSI 18: Physics and Chemistry

of Semiconductor Intertaces

Long Beach, CA

R.W. Grant, Rockwell International Science Center, P.O. Box

1085, Thousand Oaks, CA 91358 (805) $373-4219$

\section{FEBRUARY 1991}

$4-8$

Florida Advanced Materials

Chemistry Conference

Gainesville, FL

R.S. Drago, Department of

Chemistry, University of Florida,

Gainesville, Florida 32611

\section{0-15}

POLYMER 91-Polymer Materials: Preparation, Characterization and Properties

Melboume, Australia

G.B. Guises, RACI Polymer Div., P.0. Box 224, Belmont, Victoria, 3216 Australia; 05247 2695; fax 052472657

16-22

OFC-Optical Fibers Communications Conference

San Diego, CA

Lasers and Electro-Optics Society,

445 Hoes Lane, P.O. Box 1331

Piscataway, NJ 08B55-1331; (201) 562-3895; $\operatorname{tax}$ (201) 562-1571

$17-20$

14th Annual Meeting of the

Adhesion Society

Clearwater, FL

H.M. Clearfield, IBM T.J. Watson

Research Center, P.O. Box 218,

M/S 38-145, Yorktown Hejghts,

NY 10598; (914) 945-3202

17-21

TMS Annual Meeting

New Orleans, LA

TMS, 420 Commonwealth Dr.

Warrendale, PA 15086; (412) 776-

9050: $\operatorname{tax}(412) 776-3770$
28-1

MIRS Washington Materials

Forum: Superconductors and

Semiconductors

Washington, DC

Materials Research Society, 9800

Mcknight Rd., Pittsburgh, PA

15237; (412) 367-3003; fax (412) $367-4373$

\section{MARCH 1991}

4-8

42nd Pittsburgh Conference/

Exposition on Analytical Chemis-

try and Applied Spectroscopy

Chicago, IL, Pittsburgh Conter-

ence, 300 Penn Center Blvd.

Suite 332, Pittsburgh, PA 15235

11-15

International Congress on Optical

Science and Engineering

The Hague, The Nethertands

In Europe: Europtica-Services

I.C. 16 , avenue Bugeaud, 75116

Paris, France; (33.1) 45.53.26.67;

tax (33.1) 4704.25.20. In North

America/Asia: SPIE, P.O. Box 10

Bellingham, Washington, USA

98227-0010; (206) 676-3290; tax (206) 647-1445

$12-13$

Bonding and Repair of

Composites II

Zurich, Switzerland

$K$ Rovle, Rapra Technology Ltd

Shawbury, Shrewsbury, Shrop-

shire SY4 4NR, England; 44-939

250383; tax 44-939-251118

12-14

Surface Engineering: Practice and

Prospects

Adelaide, Australia

K. Brett, Dept. of Metallurgy,

South Australian Institute of

Technology, 183 Melboume St.

North Adelaide SA 5006,

Australia; 618-8-267.5466; tax

618-8-267-4031

17-21

7 th Intemational Symposium on

Halide Glasses

Lome, Victoria, Australia

D.R. MacFarlane, Centre for

Advanced Materials Technology,

Monash University, Clayton,

victoria 3168, Australia; tax 61-3.

5654998

17.22

SVC 34th Annual Technical

Conterence

Philadelphia, PA

D.M. Mattox, SVC Technical

Director, Society of Vacuum

Coaters Administrative Office, $\mathbf{4 4 0}$

Live Oak Loop, Albuquerque, NM

87122; (505) 298-7624; fax (505)

298-7942

18-22

American Physical Society

Meeting

Cincinnati, $\mathrm{OH}$

APS, 335 E. 45th St., New York

NY 10017; (212) 682.7341
24-27

3rd European Workshop on

Refractory Metals and Silicides

Stockholm, Sweden

C.S. Petersson, KTH, Box 1298

S-16428 Kista-Stockholm

Sweden; (46) 8-752 1401; fax (46) $8-7527782$

25-28

7th Oxford Conference on

Microscopy of Semiconducting

Materials

Oxford, United Kingdom

A.G. Cullis, Royal Signals \& Radar

Establishment, St. Andrews Rd.,

Malvem, Worcs WR14 3PS

United Kingdom

(See related article in

VI. XVNo. 9.)

\section{APRIL 1991}

$3-5$

19th Intemational Symposium on

Acoustical Imaging

Bochum, W. Gemany

H Emert, Ruhr-Universitat

Bochum, P.O. Box 10, 21 48/C 6

D-4630 Bochum 1, W. Germany;

234-700-2842; $\operatorname{tax}$ 234-700-2339

3-5

$3 r d$ International Symposium on

integrated Ferroelectrics

Colorado Springs, $\mathrm{CO}$

Symposium Secretary, Microelectronics Research Laboratory

University of Colorado at Colorado

Springs, P.O. Box 7150, Colorado

Springs, CO 80933-7150; (719)

593-3488; $\operatorname{tax}$ (719) 594-4257

$7 \cdot 11$

Intemational Conference on

Wear of Materials-91

Orlando, FL

L. Friedman, Meetings Dept.

ASME, 345 E. 47th St., New

York, NY 10017-2304;

(212) 705-7722

(See related article in

Vol. XIV No. 12.)

$8-9$

ISHM 1991 Joint Technology

Conterence

Dallas, Texas

R. Breck, ISHM JTC 1991, P.0.

Box 2698, Reston, VA 22090

(703) 471-0066 or (800) 232

SHM; $\operatorname{tax}(703)$ 471-1937

8-11

11th General Conference on the Condensed Matter Division of the European Physical Society

Exeter, England

The Meeting Office, The institute of Physics, 47 Belgrave Square,

London SW1X 80X, England; 44

71-235-6111; $\operatorname{tax} 44.71-2596002$

Florida Catalysis Conterence

Gainesville, FL

R. S. Drago, Department of

Chemistry, University of Florida

Gainesville, FL 3261

14-17

5th Biennial Workshop on

Organometallic Vapor Phase

Epitaxy

Panama City Beach, $\mathrm{FL}$

B. Kamperman, TMS, 420

Commonwealth Dr. Warrendale,

PA 15086-7514; (412) $776-9050$

$\operatorname{tax}(412) 776-3770$ 
$6-10$

3rd Intemational Conference on the formation of Semiconductor Interfaces

Rome, Italy

P. Perfetti, Istituto di Struttura della Materia (CNR), Via E. Ferm 38, 00044 Frascati, Italy; 39-69426335; tax 39-6-9417003

8-10

75th Anniversary Symposium on Metallography in conjunction with ASTM Stardards Meeting Atlantic City, NJ

ASTM, 1916 Race St., Philadelphia, PA 19103-1187; (215) $299-5400$

13-17

CLEO '91: Conterence on Lasers and Electro-Optics

Batimore, MD

Lasers and Electro-Optics Society, 445 Hoes Lane, P. . B. Box 1331 ,

Piscataway NJ 08855-1331; (201) 562-3895; fax (201) 562-1571

20-24

1st Intemational Workshop on Materials Processing at High

Gravity

Dubna, USSR

W.R. Wilcox, Clarkson University,

Potsdam, NY 13699-5700;

(315) 268.6446

27-31

6th International Symposium on

Intercalation Compounds

Orteans, France

F. Beguin, 1 B, ne de la Fenollerie

45071 Orleans Cedex 02. France;

38-51-53-79

\section{JUNE 1991}

$2-6$

Annual Meeting of the American Nuclear Society

Orlando, $\mathrm{FL}$

J. DeMastry, Florida P\&L Co.

P.O. Box 14000, Juno Beach, FL 33408; (407) 6943613

3-5

Polymer Technology Conference

Philadelphia, PA

D. Morrill, VPI \& SU, 1103 CRB

Blacksburg, VA 24061-0212; (703) 231.3029
10-14

Short Course on Scanning Electron Microscopy and X-ray Microanalysis

Bethlehem, PA

J.I. Goldstein, Dept. of Materials

Science and Engineering, Bldg. 5

Lehigh University, Bethlehem, PA

18015; (215) 758-5133

11-14

Cryogenic Engineering Confer-

ence and Intemational Cryogenic

Materials Conterence

Huntsville, Alabama

EW. Uman, Space Science

Laboratory, ES-63, NASA,

Marshall Space Flight Center, AL

35812; (205) 544-7721; tax (205) $544-7754$

$17-20$

th Japan Institute of Metals

Symposium on Intermetallic

Compounds

Sendai, Japan

0 . Izumi, Institute for Materials

Research, Tohoku Univ., Katahira,

Sendai 980, Japan; 81-22-227.

6200: tax 81-22-2647984

17.20

Short Course on Scanning

Electron Microscopy and X-ray

Microanalysis

Bethlehem, PA

J.I. Goldstein, Dept. of Materials

Science and Engineering, Bldg. 5,

Lehigh University, Bethlehem, PA

18015; (215) 758-5133

17-20

Short Course on Analytical

Electron Microscopy

Bethlehem, PA

J.I. Goldstein, Dept. of Materials

Science and Engineering, Bldg. 5

Lehigh University, Bethlehem, PA

18015; (215) 758-5133

18-20

1991 Dalian Intemational

Conference on Glass

Dalian, China

Conference secretary, T. Ying

Dalian Institute of Light Industry, 1

Bao Ding Street, Dalian 116001 .

Dalian, China; telex 86141 BOOTH

\section{$18-20$}

5th International SAMPE

Electronic Materials and Proc-

esses Conference

Los Angeles, CA

SAMPE Business Office, P.O. Box

2459, Covina, CA 91722; (818)

331-0616; tax (818) 332-8929

18-2

Electronic Materials Conterence

Boulder, $\mathrm{CO}$

TMS 420 Commonwealth Dr.

Warrendale, PA 15086; (412) 776

9050; fax (412) $776-3770$
1921

First Intemational Research Symposium on Environmental Eftects on Adyanced Materials San Diego, Califomia

NACE Customer Service Department, P.O. Box 218340, Houston TX 77218; (713) 492-0535, ext.

81: $\operatorname{tax}(713) 492-8254$

(See related article in

Vol. XVNo. 9.)

$19-21$

Conference on Fracture Proc-

esses in Brittle Disordered

Materials

Noordwijk, The Netherlands

Congress Office ASD, P.O. Box

54,2640 AB Pijnacker, The

Netherlands; 31-17.365356; tax 31-17-362242

20-21

Short Course on Thin Specimen

Preparation

Bethlehem, PA

J.I. Goldstein, Dept. of Materials

Science and Engineering. Bldg. 5

Lehigh University, Bethlehem, PA

18015; (215) 758-5133

24-28

6th International Conference on

Sensors and Actuators -

Transducers ' 91

San Francisco, CA

L. Reid, University Extension,

University of Califomia, Berkeley,

CA 94720; (415) 642-4151

25-27

Intemational Superconductive

Electronics Conference

Glasgow, Scotland

G.B. Donaldson, Dept. of Physics

and Applied Physics, John

Anderson Building, 107 Rot-

tenrow, University of Strathclyde,

Glasgow G4 ONG, Scottand UK:

041-552-4400; tax 041-552-2891

25-28

2nd International Conference on

Surface X-Ray and Neutron

Scattering

Bad Honnef, Germany

H. Zabel, Experimentalphysik IV

Geb. NB 4/132, Ruhr-Universität

Bochum, Posttach 1021 48, D-

4630 Bochum 1, W. Germany

49-234-700-3649; fax 49-234

$700-3643$

JULY 1991

$1-4$

Uitrasonics International ' 91

Le Touquet, France

M. Vukovojac, Butterworth

Scientific Lto., P.O. Box 63,

Westbury House, Bury St.

Guildford, Surrey GU2 5BH

United Kingdom; 483-300966; fax 483-301563

$7-12$

7th Intemational Conference on

Surface and Colloid Science

Compiegne - France

Secretariat of the 7th ICSCS, c/o

Wagons-ilts Tourisme, B.P. 244

F-92307 Levallois-Perret cedex,

France
7-20

NATO Advanced Study Institute:

Physics and Materials Science of

High $\mathrm{T}_{\mathrm{c}}$ Superconductors

Cortu Istand, Greece

R. Kossowsky, 6327 Burchtield

Ave., Pittsburgh, PA 15217; (412)

421-4408; $\operatorname{tax}(412$ ) 421-4342

14-19

19th Rare Earth Research

Conference

Lexington, $K Y$

D.D. Ensor, 19th AERC Secretary

Tennessee Technological

University, Chemistry Dept., Bax

5055, Cookeville, TN 38505

15-17

Intemational Conference on

Fracture Mechanics of Ceramics

Nagoya, Japan

M. Salai, Toyohashi University of

Technology, Tempaku-cho,

Toyohashi, 440, Japan; 0532-47.

0111: $\operatorname{tax} 0532-45-0480$

15-19

7th intemational Conterence on

Surface Modification of Metals by

Ion Beams

Washington, DC

SMMIB 91, Naval Research

Laboratory, Code 4670, Washing-

ton, OC 20375-5000; fax (202)

$767-5301$

21-26

American Crystallographic

Association Annual Meeting

Toledo, OH

M.C. Etter, Dept. of Chemistry

Univ. of Minnesota 78 Kolthofl

Hall, Minneapolis, MN 55455:

(612) $624-5217$

$22-24$

Euromat 91: 2nd European

Conterence on Advanced

Materials and Processes

University of Cambridge, United

Kingdom

Euromat 91, Conference Dept.

The Institute of Metals, 1 Cartton

House Terrace London, SW1Y

5D8, United Kingdom; 4471.

8394071; tax 44-71-8392289

$22-26$

Intemational Conference on

Materials and Mechanisms of

Superconductivity-High

Temperature Superconductors

(M²S-HTSC III)

Kanazawa, Japan

K. Kitazawa, Dept. of Industrial

Chemistry, University of Tokyo, 7

3-1 Hongo, Bunkyo-ku, Tokyo 113

Japan; 81-3-8122111, ext. 7201;

fax 81-3-8155632

293

MIRT Meeting on Epitaxia

Materials and In Situ Processing

tor Optoelectronic Devices

Newport Beach, CA

W. Rochelle, LEOS, 445 Hoes

Lane, P.O. Box 1331, Piscataway,

NJ 08855-1331; (908) 562-3895;

$\operatorname{tax}(908)$ 562-1571

291

International Conterence on Point

Defects in Glasses

Riga, Latvia

A.R. Silin, Institute of Solid State

Physics, University of Latvia, 8

Kengaraga St, 226063, Riga,

Latvia; 013-2-262933; tax 013-2

225093

$29-2$

MRISThin Film Science and

Technology in the 21st Century

Evanston, lilinois

J. Tumer, Northwestem University,

2145 Sheridan Road, $\$ 2033$

Evanston, IL 60208-3116; (708) 


\begin{tabular}{l} 
SEPTEMBER 1991 \\
\hline $1-7$ \\
Intemational Conference on \\
Magnetism \\
Edinburgh, United Kingdom \\
Meetings Officer, IOP, 47 Belgrave \\
Square, London SW1X 80X, \\
United Kingdom; 441-235-6111 \\
8-13 \\
5th Intemational Conterence on \\
I-VI Compounds \\
Okayama, Japan \\
M. Konagaj, c/0 Japan Conven- \\
tion Services, Nippon Press \\
Center Bldg., 2-2-1, Uchisaiwai- \\
cho, Chiyoda-ku, Tokyo 100, \\
Japan; 81-3-508-1213; tax 81-3- \\
508-0820 \\
15-20 \\
Symposium on Microelectronic \\
Interconnect and Integrated \\
Processing \\
San Jose, CA \\
SPIE, P.0. Box 10, Bellingham, \\
WA 98227-0010; (206) 676-3290 \\
15-20 \\
EMC '91 - First European Metals \\
Conference: Nonfermus \\
Metallurgy-Present and Future \\
Brussels, Belgium \\
J. Vereecken, Chairman of \\
Benelux Metallurgy, Vrije \\
Universiteit Brussel, Dept. \\
Metallurgy, Pleinlaan 2, 1050 \\
Brussels, Belgium; 32-2/ \\
641.32.54; tax 32-2/641.32.00 \\
\end{tabular}

17.-19

2nd Intemational Conference on

Interfacial Phenomena in

Composite Materials ' 91

Leuven, Belgium

J. Miles, Butterworth Scientific

Ltd., P.O. Box 63, Westbury

House, Bury St., Guildford,

Surrey GU2 5BH United Kingdom

44-483-300966; tax 44-483.

301563

17.19

Intemational Conference on High

Temperature Aluminides and

Intermetallics

San Diego, CA

ASM Intemational, Materials Park

$\mathrm{OH} 44073$; $\operatorname{tax}(216) 338-4634$

22-25

5th Conference on Sensors and

Their Applications

Edinburgh, United Kingdom

Meetings Office, Institute of

Physics, 47 Belgrave Square,

London SW1 80X, United

Kingdom; 44-1-235-6111

$30-2$

Specialty Polymers '91: Supramolecular Aspects of Polymer

Synthesis and Polymer Structure

Mainz, W. Germany

Conference Organizer, SP'91,

Butterworth Scientific Ltd., P.O.

Box 63, Westbury House, Bury

Street, Guildford, Sumey GU2

$5 B H$ United Kingdom; 44-483-

300966; fax 44-483-301563
30-4

Intemational Conference on lon

Sources (ICIS'91)

Darmstadt, W. Germany

B. Wolf, GSl, Postfach 110552

6100 Darmstadt 11, W. Germany

49-6151-359320; tax 49-6151-

359785

\section{OCTOBER 1991}

14-18

4th Eumpean Conference on

Applications of Surface and

Interface Analysis

Budapest, Hungary

L. Köver, Inst. of Nuclear

Research, Hungarian Academy of

Sciences, MTA ATOMKI, H-4001

Debrecen, Pf 51, Hungany

\section{0-24}

TMS Fall Meeting: Physical

Metallurgy and Materials

Cincinnati, $\mathrm{OH}$

TMS, 420 Commonwealth Dr.,

Warrendale, PA 15086; (412) 776 -

9050; fax (412) $776-3770$

21-23

Intemational Symposium on

Microelectronics

Orlando, FL

R. Breck, Intl. Society for Hybrid

Microelectronics, P.0. Box 2698 ,

Reston, VA 22090; (800) 232-

ISHM; $\operatorname{tax}(703)$ 471-1937

\section{NOVEMBER 1991 \\ 11-15 \\ 38th National AVS Symposium \\ Seattle, WA \\ American Vacuum Society, $335 \mathrm{E}$. \\ 45th St., New York, NY 10017 \\ (212) $661-9404$ \\ 17-22 \\ Intemational Conference on \\ Fusion Reactor Materials \\ Clearwater, Florida \\ P.J. Mazjasz, Metals and \\ Ceramics Division, Oak Ridge \\ National Laboratory, P.O. Box \\ 2008, Oak Ridge, TN 37831- \\ 6376; (615) 574-5082; $\operatorname{tax}(615)$ \\ MARCH 1992 \\ $16-20$ \\ APS March Meeting \\ Indianapolis, IN \\ APS, 335 E. 45th St., New York \\ NY 10017; (212) 661.9404 \\ APRIL 1992 \\ 27-2 \\ MIST Materials Research \\ Society Spring Meeting \\ San Francisco, CA \\ M. Gell, Matenials Research \\ Society, 9800 Mcknight Rd., \\ Pittsburgh, PA 15237; (412) 367 . \\ 3003; $\operatorname{tax}(412) 367-4373$}

$574-0641$

\section{DECEMBER 1991}

2.7

M.RIS Materlals Research

Society Fall Meeting

Boston, MA

M. Geil, Materials Research

Society, 9800 McKnight Rd.,

Pittsburgh, PA 15237; (412) 367

3003; $\operatorname{tax}(412)$ 367-4373

$9-12$

Polymer Waste Management

Conference

New Orleans, LA

D. Morrill, VPI \& SU, 1103 CRB,

Blacksburg, VA 24061-0212; (703)

231-3029

\section{JULY 1992}

20-24

Intemational Conference on

Martensitic Transtormations

(ICOMAT-92)

Monterey, Califomia

J. Perkins, ICOMAT-92 Secretariat,

Naval Postgraduate School, Code

ME/PS, Monterey, CA 93943;

(408) 646-2216; tax (408)

646-2761

(See related article in this issue.)

\section{Put Your Grant Proposal on Top with this MRS Video Workshop...}

\section{Grantsmanship: The Art of Funding Proposals}

Three hours of instruction from university and industry experts, including...

$\square$ Strategies for Successful Proposals

Richard Tressler, Director

Penn State Center for Advanced Materials

$\varpi$ Federal, State, and Private Agency Matching Funds

Maynard Kohler, Penn State Liaison

Federal Office of Sponsored Programs

ఐ Proposal Writing: Targeting Your Audience

Jim Hill, Senior Proposal Coordinator

HRB Singer
ఐ The Proposal Budget

Donald Fornwalt, Acting Coordinator

Research Funds in Earth and Mineral Sciences

$\square$ The Proposal Review Process

Paul Brown, Professor of Ceramic Science and Engineering

Pennsylvania State University (previously with National Institute of Standards and Technology)

Produced by the Pennsylvania State University Chapter of the Materials Research Society under the sponsorship of the MRS Educational Committee, this two-hour program was taped at a workshop at Pennsylvania State University.

Now available - on loan free of charge - from MRS. Two-week terms. VHS format. For more information, call Anne Wagner at (412) 367-3003. 